\title{
Propagación de distintos tipos funcionales de la costra biológica del suelo del desierto del Monte, Argentina
}

\author{
Vanesa García $^{1, \mathbb{D}}$; Julieta AranibaR ${ }^{1 / 2} \&$ Pablo E. Villagra ${ }^{1 / 3}$ \\ ${ }^{1}$ Instituto Argentino de Nivología, Glaciología y Ciencias Ambientales (IANIGLA). Consejo Nacional de Investigaciones \\ Científicas y Técnicas, CONICET-CCT Mendoza. Mendoza, Argentina. ${ }^{2}$ Facultad de Ciencias Exactas y Naturales, \\ Universidad Nacional de Cuyo. Mendoza, Argentina. ${ }^{3}$ Facultad de Ciencias Agrarias, Universidad Nacional de Cuyo. \\ Mendoza, Argentina.
}

RESUMEN. Las costras biológicas del suelo (CBS) son asociaciones entre cianobacterias, algas verdes, líquenes, musgos y partículas de suelo. Las costras cumplen múltiples funciones ecosistémicas tales como la fijación de nitrógeno atmosférico y la estabilización de suelos. El presente trabajo tiene como objetivo evaluar condiciones de propagación de distintos tipos funcionales de CBS (dominadas por cianobacterias, cianolíquenes, ficolíquenes y musgos) que posean potencial para la recuperación de suelos degradados. En vivero, se cultivaron diferentes tipos funcionales de CBS recolectadas en el desierto del Monte, Argentina, combinando tratamientos de siembra (CBS triturada o agregada en trozos) y fertilidad (sustrato con o sin estiércol de cabra). Las variables medidas como indicadoras de desarrollo y funcionalidad de CBS fueron la cobertura total de CBS y de cada tipo funcional, la profundidad alcanzada por la CBS, la estabilidad del suelo, y las concentraciones de amonio y fosfato. La cobertura total de CBS aumentó en 19 meses. La siembra triturada favoreció el desarrollo y funcionalidad de la CBS, en particular en las macetas sembradas con cianolíquenes, ficolíquenes y musgos. La adición de fertilizante redujo la cobertura total de CBS dominadas por ficolíquenes y musgos. La profundidad de la CBS y la estabilidad del suelo aumentaron respecto al suelo desnudo en macetas dominadas por líquenes y musgos, y estuvieron favorecidas por la siembra triturada. La concentración de nutrientes dependió del tipo funcional de CBS; en comparación con el suelo desnudo, fue menor en todos los casos. Se concluye que el cultivo de CBS en invernadero, bajo tratamientos de siembra triturada y sin fertilizante, favoreció la cobertura y profundidad de CBS y aumentó la estabilidad de los suelos. Estos resultados facilitan el diseño de estrategias de restauración de tierras secas, que incluyan a las CBS como estabilizadoras de suelos.

[Palabras clave: profundidad, estabilidad del suelo, amonio, fosfato]

\begin{abstract}
Aвstract. Propagation of biological soil crust functional types from the Monte desert, Argentina. Biological soil crusts (BSC) are associations among cyanobacteria, green algae, lichens, mosses and soil particles, which contribute to multiple ecosystem functions such as nitrogen fixation and soil stabilization. The present work aims to evaluate propagation conditions of different functional types of BSC (dominated by cyanobacteria, cyanolichens, phycolichens and mosses) potentially useful for the recovery of degraded soils. In the greenhouse, different functional types of BSC were cultivated, combining sowing treatments (crushed BSC, discrete fragments) and fertilization (substrate with or without goat manure). Response variables, indicating BSC development and functionality, were total BSC cover, each functional type cover, depth of BSC, soil stability, and ammonium and phosphate concentrations. Total BSC cover increased during the 19 months experimental period. Crushing BSC favored the development and functionality of BSC in pots dominated by cyanolichens, phycolichens and mosses. The addition of fertilizer decreased total BSC cover in pots dominated by phycolichens and mosses. BSC depth and soil stability increased in lichens and moss dominated pots, and were benefited by crushing before sowing. Nutrient concentrations were lower in soils under BSC than in bare soils. We conclude that crushing BSC before sowing and without fertilization favored BSC cover and depth and increased soil stability. These results are useful to plan restoration strategies of degraded drylands.
\end{abstract}

[Keywords: depth of biological soil crust, soil stability, ammonium, phosphate]

Editora asociada: Adriana Salvo

弨arcia@mendoza-conicet.gob.ar
Recibido: 28 de Mayo de 2020

Aceptado: 11 de Septiembre de 2020 


\section{INTRODUCCIÓN}

La costra biológica del suelo (CBS) es una asociación entre cianobacterias, algas verdes, líquenes, musgos y partículas de suelo; está presente en lugares con abundante luz y baja productividad como suelos de zonas áridas (Belnap and Lange 2001). Estas comunidades cumplen funciones ecológicas clave como aumentar la fertilidad de los suelos, ya que las cianobacterias fijan $\mathrm{N}$ atmosférico (Aranibar et al. 2003; Zhao et al. 2010). Además, la CBS favorece la germinación de plantas vasculares (Su et al. 2007) e interviene en la disminución de la erosión y estabilización de los suelos (Gao et al. 2017). La cubierta de CBS es un determinante de la estabilidad de suelos eólicos con textura arenosa en Australia y en el control de la pérdida de suelo por erosión hídrica en regiones semiáridas de China (Eldridge and Leys 2003; Zhao and Xu 2012).

En la Provincia Biogeográfica del Monte (Argentina) (Cabrera 1976), las costras biológicas contribuyen a estabilizar los suelos, a mantener la humedad y acumular $\mathrm{N}$ en micrositios bajo arbustos, y a regular la infiltración al sustituir plantas vasculares en interparches (Garibotti et al. 2018). En el Monte central se realizan varias prácticas de uso de la tierra, tales como la tala, el desmonte para la actividad petrolera y la ganadería, que es la actividad dominante. Esta última, según la intensidad y el tipo de suelo, puede causar degradación local, con pérdidas de suelos, de cobertura vegetal y de productividad, y desertificación (Villagra et al. 2009; Goirán et al. 2012; Villagra and Álvarez 2019). El pastoreo también afecta de forma negativa a las comunidades de CBS en el desierto del Monte, disminuye su cobertura, modifica su distribución espacial y restringe su crecimiento a suelos protegidos del pisoteo por arbustos o madera muerta (Kröpfl et al. 2007; Gómez et al. 2012; Tabeni et al. 2014; García et al. 2015).

La funcionalidad y la importancia ecológica de las CBS dependen tanto del tipo de organismo fotosintético dominante - ya sean líquenes, musgos o cianobacterias - como de la morfología y la estructura tridimensional que forman las comunidades (Bowker et al. 2011; Pietrasiak et al. 2013). Por lo tanto, es común clasificar las comunidades según la morfología y el tipo funcional que las domina, como costras dominadas por cianobacterias, cianolíquenes gelatinosos y escamulosos, ficolíquenes escamulosos y musgos (Bowker et al. 2006; Read et al. 2014). Por ejemplo, las costras dominadas por cianobacterias con heterocistos y cianolíquenes gelatinosos, como el género Collema, son capaces de fijar nitrógeno atmosférico (Belnap 2002). En cambio, las costras dominadas por musgos son capaces de retener mayor cantidad de sedimento y absorber mayor cantidad de agua (Mallen-Cooper and Eldridge 2016).

Con el objetivo de revertir los procesos de degradación ocasionados por el excesivo uso de estas tierras, en la Argentina se crearon áreas protegidas y en los últimos años se están llevando a cabo proyectos de reforestación y restauración de sitios degradados (Dalmasso 2010; Villagra et al. 2010; Bessega et al. 2019). La mayoría de estos trabajos de restauración se realizan en zonas áridas e incluyen principalmente la revegetación con plantas vasculares, como especies de arbustos nativos del desierto de Monte en la Argentina, Capparis atamisquea, Larrea cuneifolia, Atriplex Lampa y arbóreas como Prosopis flexuosa y Prosopis chilensis (Dalmasso 2010; Fernández et al. 2016; Segui 2019). Sin embargo, estos proyectos orientados a la recuperación de suelos no incluyen a la CBS en su sistema de estudio, tal vez debido a la escasez de conocimiento local sobre estos organismos. En los últimos años, en países como Estados Unidos, China y España entre otros, se comenzaron a estudiar técnicas de inoculación y trasplante de la CBS para implementarla en la restauración de ecosistemas (Chen et al. 2006; Cole et al. 2010; Antoninka et al. 2016; Chiquoine et al. 2016). Estas técnicas incluyen el cultivo en invernadero de cianobacterias, líquenes y musgos, bajo distintos regímenes de hidratación y fertilización, buscando recuperar funciones asociadas a las costras biológicas y optimizar condiciones de rendimiento y producción de estos organismos (Maestre et al. 2006; Antoninka et al. 2016). En otros casos se realizaron experimentos sobre suelos desérticos inicialmente cultivando cianobacterias en invernadero y luego inoculándolas, mediante rociado (Wang et al. 2009), o directamente trasplantando fragmentos de CBS (Chiquoine et al. 2016) para evaluar el efecto en la colonización, el desarrollo y la mejora de las propiedades del suelo. En ecosistemas muy disturbados, organismos de la CBS como cianobacterias se comportan como colonizadores, y por su capacidad de agregar las partículas del suelo actúan como facilitadores para el establecimiento de plantas vasculares (Eldridge et al. 2006; Williams et al. 2012). 
Debido a la necesidad de restauración de suelos degradados del Monte en la Argentina y a la multifuncionalidad de la CBS, el presente trabajo tiene como objetivo identificar métodos de propagación de distintos tipos funcionales de CBS de la región, que posean potencial para restaurar ecosistemas degradados, mediante ensayos de propagación en vivero, fertilización de suelo e implementación de distintas técnicas de siembra. Se hipotetiza que la desagregación de cianobacterias, líquenes y musgos, y el agregado de fertilizantes naturales al sustrato antes de la siembra aceleran el desarrollo y la profundidad de la CBS, y recuperan la funcionalidad (i.e., estabilidad, fertilidad) de suelos debido a que aumenta la reproducción vegetativa de estos organismos. Se espera encontrar: a) que los grupos de organismos de la CBS alcancen una mayor cobertura cuando se siembran de forma triturada, b) que los suelos con agregado de fertilizante tengan mayor cobertura de CBS que suelos sin fertilizar, c) que los tipos funcionales de CBS inoculada se diferencien en la profundidad y la estabilidad que dan al suelo, que los musgos brinden mayor estabilidad, d) que la cobertura de CBS alcanzada 19 meses después de la inoculación se relacione positivamente con la estabilidad de suelos y el contenido de nutrientes.

\section{Materiales y Métodos}

Para determinar métodos propagación y desarrollo de la costra biológica del suelo (CBS) se realizó un ensayo en invernadero, donde se cultivaron en macetas, distintos tipos de CBS, dominadas por cianobacterias, cianolíquenes, ficolíquenes y musgos, durante 19 meses desde mayo del 2017 (otoño) a noviembre del 2018 (primavera).

\section{Recolección del material}

Con el fin de minimizar el impacto negativo sobre las comunidades de CBS, los fragmentos de CBS y el sustrato a utilizar fueron colectados de los bordes de caminos que atraviesan la reserva de la Biosfera de Nacunán, Mendoza, Argentina. La recolección de material se realizó durante los años 2015 y 2016, seleccionando muestras claramente dominadas por un tipo funcional (que alcanzaran una cobertura cercana al $90-100 \%$, estimada visualmente) y posteriormente en el laboratorio se realizó la clasificación de las CBS en los distintos tipos funcionales dominantes en cada muestra: cianobacterias, cianolíquenes gelatinosos, ficolíquenes escamulosos y musgos. Se seleccionaron estos tipos funcionales para representar distintos grupos biológicos (cianobacterias, musgos, líquenes), simbiontes (ficolíquenes y cianolíquenes, potencialmente fijadores de N) y morfología (plana, tridimensional), que se espera tengan distintos efectos en los ciclos de agua, $\mathrm{C}$ y N, y en la retención de partículas de suelo y su estabilización. Los géneros más frecuentes de cada tipo funcional se determinaron mediante observaciones en microscopio y lupa, y el uso de claves (Casas et al. 2006; Komárek et al. 2015; Nash III et al. 2002). Se observaron las cianobacterias Scytonema y Microcoleus, cianolíquenes Enchylium, ficolíquenes Placidium y musgos Tortula, Bryum y Pseudocrossidium.

\section{Diseño experimental}

Para evitar el sobrecalentamiento y que lleguen las precipitaciones a las macetas, el invernadero se cubrió con nylon polietileno de 150 micrones y con una malla (media sombra) de $65 \%$. La radiación fotosintéticamente activa (RFA) se midió con radiómetro con barra integradora de $1 \mathrm{~m}$ (radiómetro Li-COR modelo LI-185 A Lincoln, Nebraska, USA) bajo la malla y en áreas expuestas. Al mediodía solar en días despejados de verano, la RFA tuvo un valor promedio de $2275 \mu \mathrm{mol} \mathrm{m} \mathrm{m}^{-2} \mathrm{~s}^{-1}$ y la malla media sombra junto con el nylon proporcionaron niveles de $65.3 \%$ de sombra $\left(787.5 \mu \mathrm{mol} \mathrm{m}^{-2} \mathrm{~s}^{-1}\right)$. Al mediodía solar en días despejados de invierno, la RFA tuvo un valor promedio de $1050 \mu \mathrm{mol} \mathrm{m}^{-2} \mathrm{~s}^{-1}$. La malla media sombra junto con el nylon brindaron niveles de $62 \%$ de sombra $\left(400 \mu \mathrm{mol} \mathrm{m}^{-2} \mathrm{~s}^{-1}\right)$. En la reserva Ñacuñán se han registrado en micrositios bajo Prosopis flexuosa valores de radiación de 590 $\mu \mathrm{mol} \mathrm{m} \mathrm{m}^{-2} \mathrm{~s}^{-1}$ (Sartor 2015).

La temperatura en el invernadero se registró cada 15 minutos, desde mayo del 2017 a marzo del 2018, con un sensor de temperatura HOBO. En los meses de invierno se registró una temperatura promedio de $11.54{ }^{\circ} \mathrm{C}$, con una mínima de $-5.24^{\circ} \mathrm{C}$. En los meses de verano se registró una temperatura promedio de 26.53 ${ }^{\circ} \mathrm{C}$, con una máxima de $47.76^{\circ} \mathrm{C}$. En el mismo período del desarrollo del ensayo entre los años 2017 y 2018, Nacuñán registró en los meses de invierno una temperatura media de $9.97^{\circ} \mathrm{C}$ y una mínima de $-8.2^{\circ} \mathrm{C}$. En los meses de verano se registró una temperatura media de $25{ }^{\circ} \mathrm{C}$, con una máxima de $44.1^{\circ} \mathrm{C}$ [https: //tinyurl.com/y2arxb8v]. 
Para el ensayo se utilizaron macetas plásticas negras sopladas de $10.5 \mathrm{~cm}$ de diámetro superior, $8.5 \mathrm{~cm}$ de altura y $8 \mathrm{~cm}$ de diámetro de base. La CBS fue cultivada bajo dos tratamientos de siembra (triturada, o agregando trozos de $1 \mathrm{~cm}^{2}$ ) y fertilización (agregado de estiércol de cabra al sustrato o sin estiércol). Para la siembra en trozos se tomaron muestras de $4 \mathrm{~cm}^{2}$, se cortaron en cuatro secciones de $1 \mathrm{~cm}^{2}$ y se colocaron distribuidos uniformemente en la maceta. Para el tratamiento de CBS triturada se seleccionó una sección equivalente de CBS $\left(4 \mathrm{~cm}^{2}\right)$, se trituró con mortero, se distribuyó homogéneamente en la superficie de la maceta y se estabilizó con un riego inicial. La fertilización se realizó mediante el agregado de $2 \mathrm{~g}$ de estiércol al sustrato, homogeneizado antes de incorporarlo a la maceta. Esta cantidad se calculó en base a concentraciones de $\mathrm{N}$ y $\mathrm{P}$ de estiércol de cabra (Gichangi and Mnkeni 2009; Irshad et al. 2013; Mupondi et al. 2006) y suelos de Ñacuñán (Corvalán et al. 2018), para aumentar la concentración aproximadamente al doble. Se consideraron 5 repeticiones de cada tipo funcional para cada una de las cuatro combinaciones de tratamiento de siembra y fertilización. Además, para cada combinación de tratamiento de siembra y fertilización se establecieron macetas controles que contenía suelo desnudo (5 repeticiones).

El sistema de riego se llevó a cabo mediante capilaridad con agua destilada. En el interior de las macetas se colocó un círculo de esponja de $9 \mathrm{~cm}$ diámetro y $2 \mathrm{~cm}$ de espesor. Luego se pesaron $250 \mathrm{~g}$ de sustrato, se agregaron por encima de la esponja y por último se sembró la CBS. Luego, las macetas armadas se dispusieron en bloques en grupos de 12 en

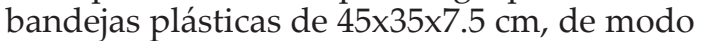
tal que, al agregarse agua a las bandejas, la esponja absorbiera agua y por capilaridad ésta ascienda hasta la superficie de las macetas, humedeciendo las CBS. El riego se realizó semanalmente, manteniendo la humedad cercana a la capacidad de campo de la maceta, calculada en macetas sin inocular.

\section{Variables respuesta determinadas}

Al concluir el ensayo se midieron las siguientes variables relacionadas con el crecimiento y desarrollo de la costra: cobertura total de CBS, cobertura de cada tipo de CBS, profundidad de la CBS, estabilidad del suelo y concentraciones de amonio y fosfato.

Cobertura de CBS. Se estimó el porcentaje de cobertura final total de CBS obtenida en cada maceta y la cobertura de cada tipo funcional (cianobacterias, cianolíquenes, ficolíques y musgos), utilizando grillas circulares del tamaño de las macetas, dividas en cuadrículas de $1.5 \times 1.5 \mathrm{~cm}$, en las que se contó el número de puntos interceptados por cada tipo funcional de CBS, con un total de 44 puntos por maceta.

Estabilidad y profundidad de la costra. Al finalizar el ensayo se utilizó una pequeña espátula de aluminio para levantar de cada maceta suavemente tres fragmentos cuadrados de suelo de 8-10 mm de lado, que no incluyera el trozo de CBS implantado en el caso del tratamiento de CBS sin triturar. Para cada muestra, mediante la utilización de un calibre se calculó la profundidad que alcanzaron los organismos que componen la CBS, considerando filamentos de cianobacterias y rizoides de musgos. Luego, cada una de esas muestras se clasificó según valores de un índice de estabilidad cualitativa (de 1 a 6: estabilidad más baja a más alta) utilizando un kit de estabilidad de suelo de USGS (United States Geological Survey Soil Stability Kit) (Herrick et al. 2001), basado en la desintegración del suelo ante distintas intensidades de disturbio mecánico (inmersión en agua y agitación). La profundidad y la estabilidad de los tres fragmentos se promediaron para obtener un valor por cada maceta.

Determinaciones de nutrientes en el suelo. Inicialmente, se retiraron y excluyeron de los análisis de funcionalidad los trozos de CBS de $1 \mathrm{~cm}^{2}$ sembrados al comenzar el ensayo para detectar únicamente el efecto de la CBS que creció durante los 19 meses. Luego, para las determinaciones de nutrientes disponibles se retiró y utilizó el cm más superficial de suelo de cada maceta. El suelo se homogeneizó con un mortero. El amonio se extrajo con cloruro de potasio ( $5 \mathrm{~g}$ en $40 \mathrm{~mL}$ de $\mathrm{KCl} 2 \mathrm{~N}$ ), y su concentración se determinó con el método de azul de indofenol (Weatherburn 1967). El fósforo disponible se extrajo con bicarbonato de sodio ( $2 \mathrm{~g}$ en $40 \mathrm{~mL}$ de bicarbonato de sodio $0.5 \mathrm{M}$ y $1 \mathrm{~g}$ de carbón activado) y se determinó con el método de Olsen y Bray (Okalebo et al. 1993). Las concentraciones de amonio y fosfato se obtuvieron mediante espectrofotometría a 636 y 880 nm, respectivamente, utilizando un espectrofotómetro HACH DR2800.

\section{Análisis estadístico}

Los datos se analizaron utilizando paquetes estadísticos fitdistrplus, glm y 
MuMIn, corrplot en el entorno estadístico R (RStudio Team 2015). Inicialmente se ajustó la distribución de los datos mediante gráficos Q-Q plot realizados por el paquete fitdistrplus (Delignette-Muller and Dutang 2015), comparando entre las distribuciones normal, lognormal y gamma y se comprobó que los datos seguían distribución normal y log normal. Las variables respuesta fueron cobertura total de CBS, cobertura por tipo funcional, estabilidad del suelo, profundidad de CBS, amonio y fosfato. Estas se modelaron en función de las variables explicativas categóricas: tipo funcional, tipo de siembra y fertilización. Se utilizó el paquete MuMin (inferencia multimodelo) (Barton 2018) para la comparación de modelos que incluían distintas combinaciones de factores fijos para cada variable respuesta, considerando los criterios de AICc (criterio de información de Akaike para muestras pequeñas) (Wong and Li 1998) y peso. Los modelos con dAICc menor a dos se utilizaron para inferir los factores fijos que afectaron a las variables respuesta. También se calcularon los dAICc entre los mejores modelos y el modelo nulo, y sólo si estos fueron mayores a dos se consideraron en los análisis. Con el mejor modelo (menor $\mathrm{dAIC}$ ), se obtuvieron los estimadores, que indican magnitud y dirección, y el valor $\mathrm{P}$. Se calculó la matriz de correlación para las variables cuantitativas cobertura total de CBS, estabilidad del suelo, profundidad de CBS, amonio y fosfato utilizando la función cor en RStudio y se representó gráficamente mediante el paquete corrplot (Wei and Simko 2017).

\section{Resultados}

\section{Cobertura total de costras biológicas del suelo} (CBS)

Los mejores modelos (dAICc menor a 2) para la cobertura total de CBS incluyeron a los factores fijos tipo funcional y los tratamientos de fertilización y siembra, sin interacciones (Tabla 1). La cobertura total de CBS aumentó en macetas donde se sembraron CBS dominadas por ficolíquenes, cianolíquenes y musgos, con respecto al suelo desnudo (Sd) (Figura 1). Las macetas sembradas con cianobacterias tuvieron generalmente coberturas totales inferiores al $20 \%$ y similares a las alcanzadas en macetas control, a excepción de cianobacterias sembradas en trozos y sin fertilizar que consiguieron coberturas levemente mayores, de $22 \%$ (Figura 1). El tratamiento de siembra de CBS mejoró el modelo (Tabla1) y aumentó la cobertura total de CBS cuando los organismos se sembraron triturados (Tabla 2).

La fertilización tuvo un efecto negativo en la cobertura total de CBS en macetas sembradas con ficolíquenes y musgos (Tabla 3). Además, la siembra de CBS triturada aumentó la cobertura total en estas macetas ya que mostró tendencia positiva comparándola con siembra en trozos (Tabla 3). En las macetas inoculadas con cianobacterias, los tratamientos de fertilización y siembra triturada tuvieron efecto nulo sobre la cobertura total de CBS (Tabla 3). Para macetas sembradas con cianolíquenes la cobertura total de costras fue levemente mayor con el agregado de

Tabla 1. Modelos lineales generalizados ( $\mathrm{glm}$ ) para cada variable respuesta. $\mathrm{m} 1$ : modelo de mayor peso Akaike y menor AICc; m2: segundo modelo en mayor peso; m0: modelo nulo. Además, se muestra el dAICc entre los dos modelos y los dAICc del mejor modelo con el modelo nulo (Tabla 1). Los dAICc entre los modelos m1 y m2 fueron, en todos los casos, menores a 2, por lo que se consideró que ambos modelos explican los datos.

Table 1. Generalized linear models (glm) for each response variable. $\mathrm{m} 1$ : model with the highest Akaike weight and lowest AICc; m2: second best model; m0: null model. The dAICc between the two models is also shown. The dAICc between $\mathrm{m} 1$ and $\mathrm{m} 2$ were, in all cases, less than 2 , so both models explain the data.

\begin{tabular}{|c|c|c|c|c|c|}
\hline Variable de respuesta & "Mejor" modelo m1 & $\mathrm{m} 2$ & dAICc m2-m1 & Peso m1 & $\mathrm{dAICc} \mathrm{m} 1-\mathrm{m} 0$ \\
\hline $\begin{array}{l}\text { Cobertura costra biológica } \\
\text { del suelo (CBS) }\end{array}$ & $\begin{array}{c}\text { Tipo funcional + } \\
\text { fertilización + siembra }\end{array}$ & $\begin{array}{l}\text { Tipo funcional + } \\
\text { fertilización }\end{array}$ & 0.11 & 0.29 & 53.98 \\
\hline Estabilidad & Tipo funcional + siembra & Tipo funcional & 1.39 & 0.40 & 54.67 \\
\hline Profundidad & Tipo funcional + siembra & Tipo funcional & 0.17 & 0.27 & 34.90 \\
\hline Amonio & Tipo funcional & $\begin{array}{l}\text { Tipo funcional }+ \\
\text { siembra }\end{array}$ & 0.68 & 0.32 & 54.94 \\
\hline Fosfato & $\begin{array}{l}\text { Tipo funcional + } \\
\text { fertilización }\end{array}$ & $\begin{array}{c}\text { Tipo funcional }+ \\
\text { fertilización + siembra }\end{array}$ & 0.23 & 0.53 & 108.12 \\
\hline
\end{tabular}




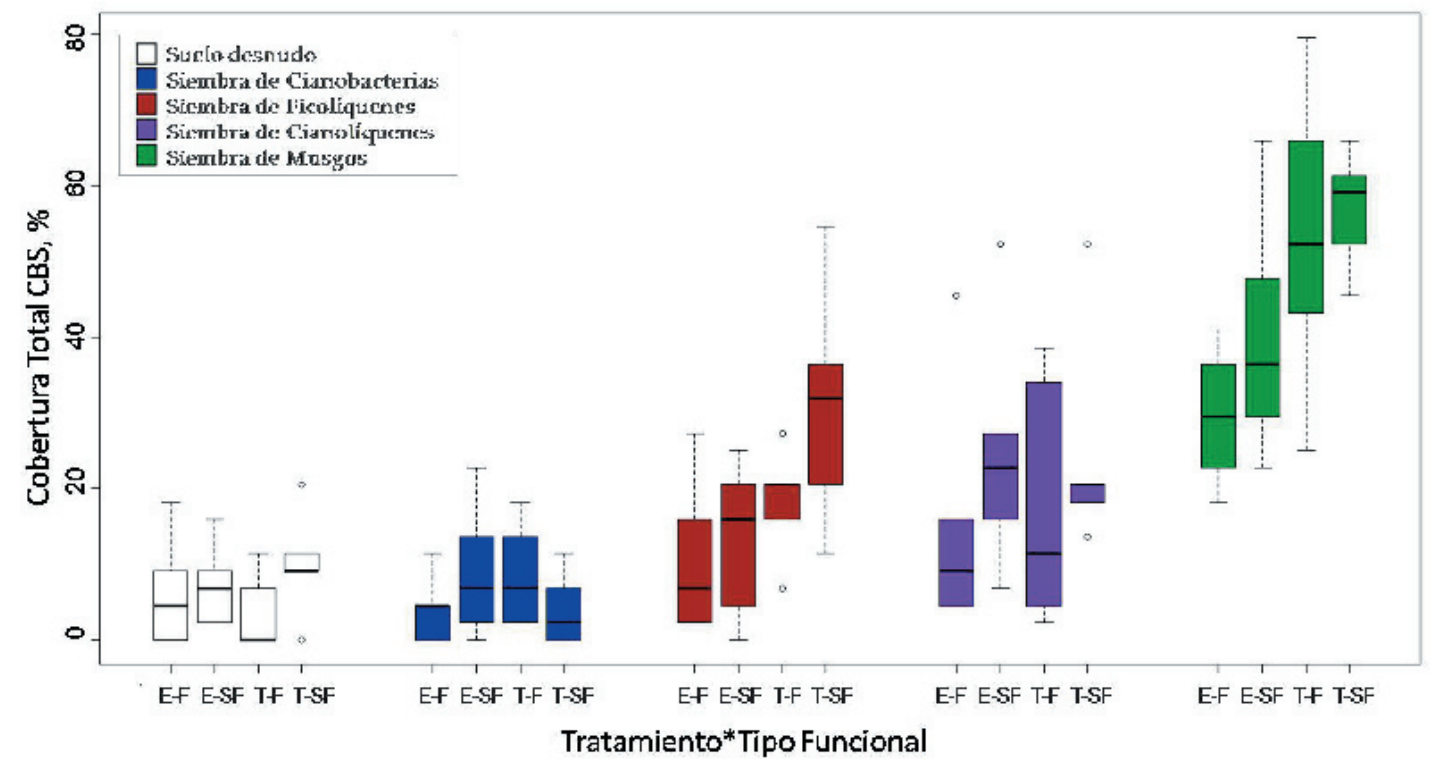

Figura 1. Cobertura total de costras biológicas del suelo (CBS) a tiempo final en macetas inoculadas con los diferentes tipos funcionales de CBS para cada tratamiento. E-F: CBS en trozos, fertilizado; E-SF: CBS en trozos, sin fertilizar; T-F: CBS triturada, fertilizado; T-SF: CBS triturada, sin fertilizar.

Figure 1. Total biological soil crust (BSC) cover for pots inoculated with different functional types for each treatment. E-F: BSC in discrete fragments, fertilized; E-SF: BSC in discrete fragments, unfertilized; T-F: crushed BSC, fertilized; T-SF: crushed BSC, unfertilized.

Tabla 2. Efecto de los tipos funcionales, tipo de siembra y fertilización sobre la cobertura total de costras biológicas del suelo (CBS). Se muestra el valor de los estimadores y de $P$. El intercepto es el suelo desnudo sin fertilizar y la siembra en trozos.

Table 2. Effect of biological soil crust (BSC) functional types, sowing, and fertilization on total BSC cover. Estimates and $P$ values are shown. The intercept refers to unfertilized bare soil and sowing with discrete fragments.

\begin{tabular}{lcc}
\hline & $\begin{array}{c}\text { Estimador inversa log, } \\
\% \text { CBS }\end{array}$ & $\operatorname{Pr}(>|\mathrm{t}|)$ \\
\hline Intercepto & 4.97 & $<0.0001$ \\
Cianobacteria & 0.97 & 0.9357 \\
Ficoliquen & 2.88 & 0.0003 \\
Cianoliquen & 3.46 & $<0.0001$ \\
Musgo & 8.90 & $<0.0001$ \\
Fertilización & 0.70 & 0.0540 \\
Triturado & 1.32 & 0.1299 \\
& & \\
\hline
\end{tabular}

fertilizante y el tratamiento de siembra no influyó en la cobertura total (Tabla 3).

\section{Respuesta de cada tipo funcional a los tratamientos}

Se analizó la cobertura a tiempo final de cada tipo funcional en los cinco tipos de macetas, suelo desnudo y sembradas con cianobacteria, ficolíquenes, cianolíquenes y musgos. Todas las macetas tuvieron presencia de al menos tres de los cuatro tipos funcionales considerados (Figura 2). Las cianobacterias crecieron en todos los tipos de macetas, aunque alcanzaron las mayores coberturas, de $20 \%$, en macetas donde se sembraron cianolíquenes. Los cianolíquenes se encontraron mayormente en macetas sembradas con musgos y con cianolíquenes, alcanzando coberturas de hasta $7 \%$. El musgo fue el tipo funcional que mejor se desarrolló, estando presente en todas las macetas y alcanzaron coberturas de hasta $60 \%$. Tanto para macetas sembradas con ficolíquenes, cianolíquenes y musgos, los organismos que alcanzaron mayor cobertura fueron los musgos. Por el contrario, los ficolíquenes sólo estuvieron presentes en macetas donde se sembró este tipo funcional y no superaron coberturas del 8\% (Figura 2).

\section{Estabilidad del suelo y profundidad de la costra}

Para la estabilidad el mejor modelo incluyó tipo funcional y tratamiento de siembra (Tabla 1). La siembra de CBS triturada tuvo un efecto positivo con respecto a macetas donde se sembró CBS en trozos (Tabla 4). La estabilidad del suelo resultó en orden creciente desde macetas control (suelo desnudo) a macetas sembradas con cianobacterias, ficolíquenes, cianolíquenes y musgos. La 
Tabla 3. Resultados del mejor modelo que explica cobertura total de costras biológicas del suelo (CBS) por tipo funcional. Los modelos incluidos tienen mayor peso y dAICc $<2$ (dAICc entre el mejor modelo y el siguiente de mayor peso). Además, se muestra el valor de los estimadores de cada modelo y las recomendaciones para la propagación de CBS derivadas de los modelos (Manejo). Variables explicativas: tipo de siembra (en trozos y triturada) y fertilización (con y sin agregado de estiércol).

Table 3. Results of the best model that explains total biological soil crust (BSC) cover by functional type are presented. Models with the highest Akaike weight and $\mathrm{dAIC}<2$ (dAICc between the two models) are shown. Estimate values and recommended strategies for CBS propagation (Manejo) are also shown. Explanatory variables: type of sowing (by discrete fragments and crushed) and fertilization (with and without the manure).

\begin{tabular}{|c|c|c|c|c|c|c|}
\hline Cobertura total de CBS & $\begin{array}{l}\text { Modelos } \\
\text { Mejor ajuste dAICc }<2\end{array}$ & dAICc & Peso & Estimadores & & Manejo \\
\hline $\begin{array}{l}\text { Macetas sembradas } \\
\text { con Cianobacterias }\end{array}$ & Nulo & 0.00 & 0.628 & Intercepto & 1.50 & Sin conclusión \\
\hline \multirow[t]{2}{*}{$\begin{array}{l}\text { Macetas sembradas } \\
\text { con Ficolíquenes }\end{array}$} & Siembra & 0.00 & 0.435 & $\begin{array}{l}\text { Intercepto } \\
\text { Siembra triturada }\end{array}$ & $\begin{array}{l}12.04 \\
12.5\end{array}$ & $\begin{array}{l}\text { Sin fertilizar/ } \\
\text { triturar }\end{array}$ \\
\hline & Fertilización + siembra & 0.91 & 0.276 & $\begin{array}{l}\text { Intercepto } \\
\text { Siembra triturada } \\
\text { Fertilizado }\end{array}$ & $\begin{array}{l}15.79 \\
12.50 \\
-7.50\end{array}$ & \\
\hline \multirow[t]{2}{*}{$\begin{array}{l}\text { Macetas sembradas } \\
\text { con Cianolíquenes }\end{array}$} & Fertilización & 0.00 & 0.447 & $\begin{array}{l}\text { Intercepto } \\
\text { Fertilizado }\end{array}$ & $\begin{array}{l}21.11 \\
0.50\end{array}$ & Fertilizar \\
\hline & Nulo & 0.45 & 0.356 & Intercepto & 2.71 & \\
\hline \multirow[t]{2}{*}{$\begin{array}{l}\text { Macetas sembradas } \\
\text { con Musgos }\end{array}$} & Siembra & 0.00 & 0.630 & $\begin{array}{l}\text { Intercepto } \\
\text { Siembra triturada }\end{array}$ & $\begin{array}{l}35.00 \\
20.00\end{array}$ & $\begin{array}{l}\text { Sin fertilizar/ } \\
\text { triturar }\end{array}$ \\
\hline & Fertilización + siembra & 1.74 & 0.264 & $\begin{array}{l}\text { Intercepto } \\
\text { Siembra triturada } \\
\text { Fertilizado }\end{array}$ & $\begin{array}{l}38.63 \\
20.00 \\
-7.27\end{array}$ & \\
\hline
\end{tabular}

Tabla 4. Efecto de los tipos funcionales y tipo de siembra sobre la estabilidad del suelo y profundidad de las costras biológicas del suelo (CBS). Se muestra el valor de los estimadores y de P. El intercepto hace referencia a suelo desnudo y siembra en trozos de CBS.

Table 4. Effect of biological soil crust (BSC) functional types and sowing on soil stability and BSC depth. Estimates and $\mathrm{P}$ values are shown. The intercept refers to bare soil and sowing with discrete fragments.

\begin{tabular}{lcccc}
\hline & $\begin{array}{c}\text { Estabilidad } \\
\text { Estimador } \\
\text { inversa log }\end{array}$ & $\operatorname{Pr}(>|\mathrm{t}|)$ & $\begin{array}{c}\text { Profundidad }(\mathrm{mm}) \\
\text { Estimador }\end{array}$ & $\operatorname{Pr}(>|\mathrm{t}|)$ \\
\hline Intercepto & 2.80 & $<0.0001$ & 0.29 & $<0.0001$ \\
Cianobacteria & 1.06 & 0.4253 & 0.13 & 0.2607 \\
Ficoliquen & 1.36 & $<0.0001$ & 0.46 & 0.0002 \\
Cianoliquen & 1.39 & $<0.0001$ & 0.61 & $<0.0001$ \\
Musgo & 1.85 & $<0.0001$ & 0.72 & $<0.0001$ \\
Triturado & 1.09 & 0.0628 & 0.11 & 0.1272 \\
\hline
\end{tabular}

diferencia de estabilidad más notoria entre los tratamientos de siembra (triturada y en trozos) se observó en cianobacterias y en musgos, en los que algunas macetas con siembra triturada alcanzaron el máximo nivel de estabilidad de la escala considerada (Figura 3).

El modelo que mejor explicó la profundidad de la CBS incluyó tipo funcional y siembra (Tabla 1). La siembra de CBS dominada por todos los tipos funcionales, excepto cianobacterias, aumentó los milímetros de profundidad que alcanzaron las CBS con respecto a macetas control. La profundidad alcanzada por los distintos tipos funcionales aumentó en orden creciente en macetas dominadas por cianobacterias, ficolíquenes y con valores similares en cianolíquenes y musgos (Figura 3). El tipo de siembra triturada aumentó levemente la profundidad de la CBS en relación a macetas donde se colocaron trozos de CBS (Tabla 4).

\section{Nutrientes en el suelo}

Para la concentración de amonio, el mejor modelo es el que incluyó únicamente el factor tipo funcional de CBS (Tabla1). Las macetas 

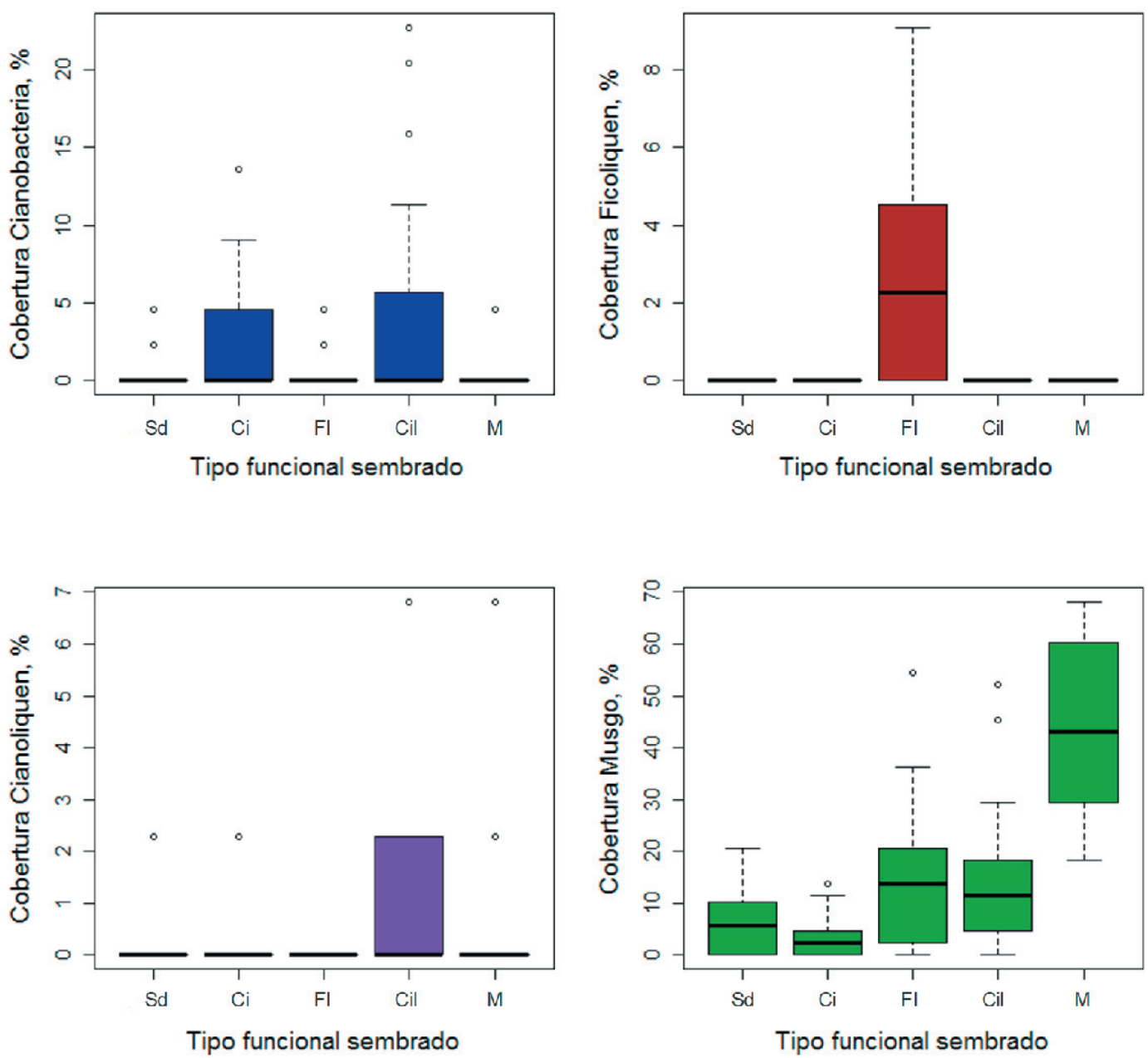

Figura 2. Cobertura de cada tipo funcional de costras biológicas del suelo (CBS) en macetas inoculadas con los diferentes tipos funcionales (Ci: cianobacterias; Fl: ficolíquenes; Cil: cianolíquenes; M: musgos) y suelos desnudos (Sd).

Figure 2. Cover of each functional type of biological soil crust (BSC) in pots inoculated with the different functional types. (Ci: cyanobacteria; Fl: phycolichens; Cil: cyanolichens; M: mosses) and bare soils (Sd).

Tabla 5. Efecto de los tipos funcionales y la fertilización sobre la concentración de $\mathrm{N}^{-} \mathrm{NH}_{4}^{+}$y $\mathrm{P}-\mathrm{PO}_{4}{ }^{3-}$. Se muestran el

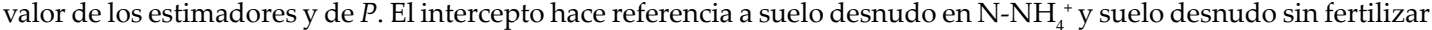
en $\mathrm{PO}_{4}=$.

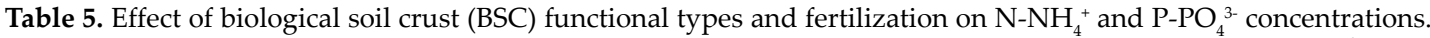
Estimates and $P$ values are shown. The intercept refers to bare soil in $\mathrm{N}_{-} \mathrm{NH}_{4}^{+}$and unfertilized bare soil in $\mathrm{PO}_{4}^{3-}$.

\begin{tabular}{|c|c|c|c|c|}
\hline & $\begin{array}{l}\text { Amonio }\left(\mathrm{N}-\mathrm{NH}_{4}^{+}\right) \\
\text {Estimador }\end{array}$ & $\operatorname{Pr}(>|t|)$ & $\begin{array}{c}\text { Fosfato }\left(\mathrm{P}_{-} \mathrm{PO}_{4}{ }^{3-}\right) \\
\text { Estimador inversa log }\end{array}$ & $\operatorname{Pr}(>|t|)$ \\
\hline Intercepto & 32.47 & $<0.0001$ & 9.32 & $<0.0001$ \\
\hline Cianobacteria & -12.53 & $<0.0001$ & 0.98 & 0.8440 \\
\hline Ficoliquen & -3.74 & 0.0498 & 0.90 & 0.2691 \\
\hline Cianoliquen & -12.19 & $<0.0001$ & 0.88 & 0.1567 \\
\hline Musgo & -13.53 & $<0.0001$ & 0.76 & 0.0024 \\
\hline Fertilización & - & - & 2.20 & $<0.0001$ \\
\hline
\end{tabular}



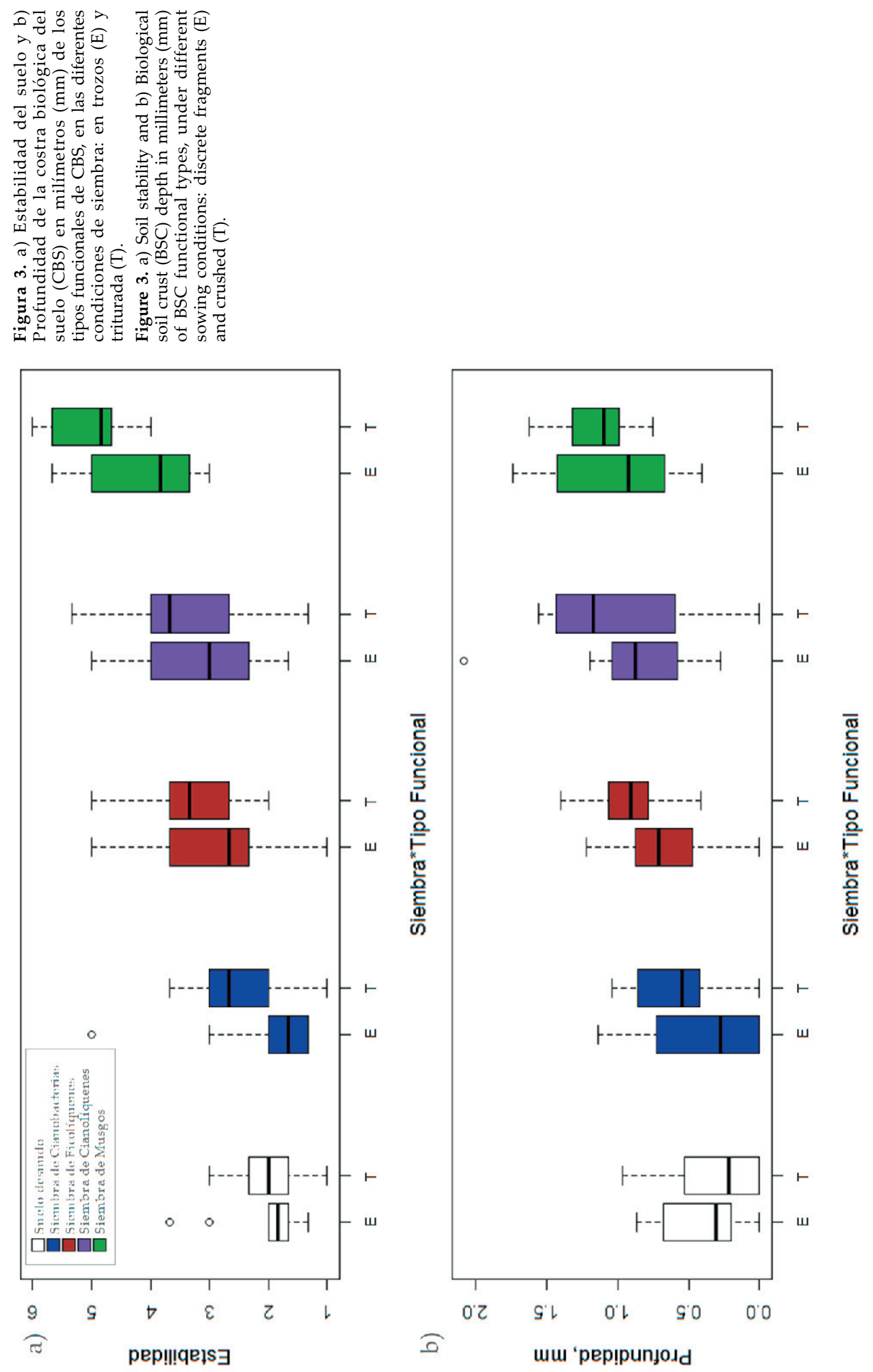

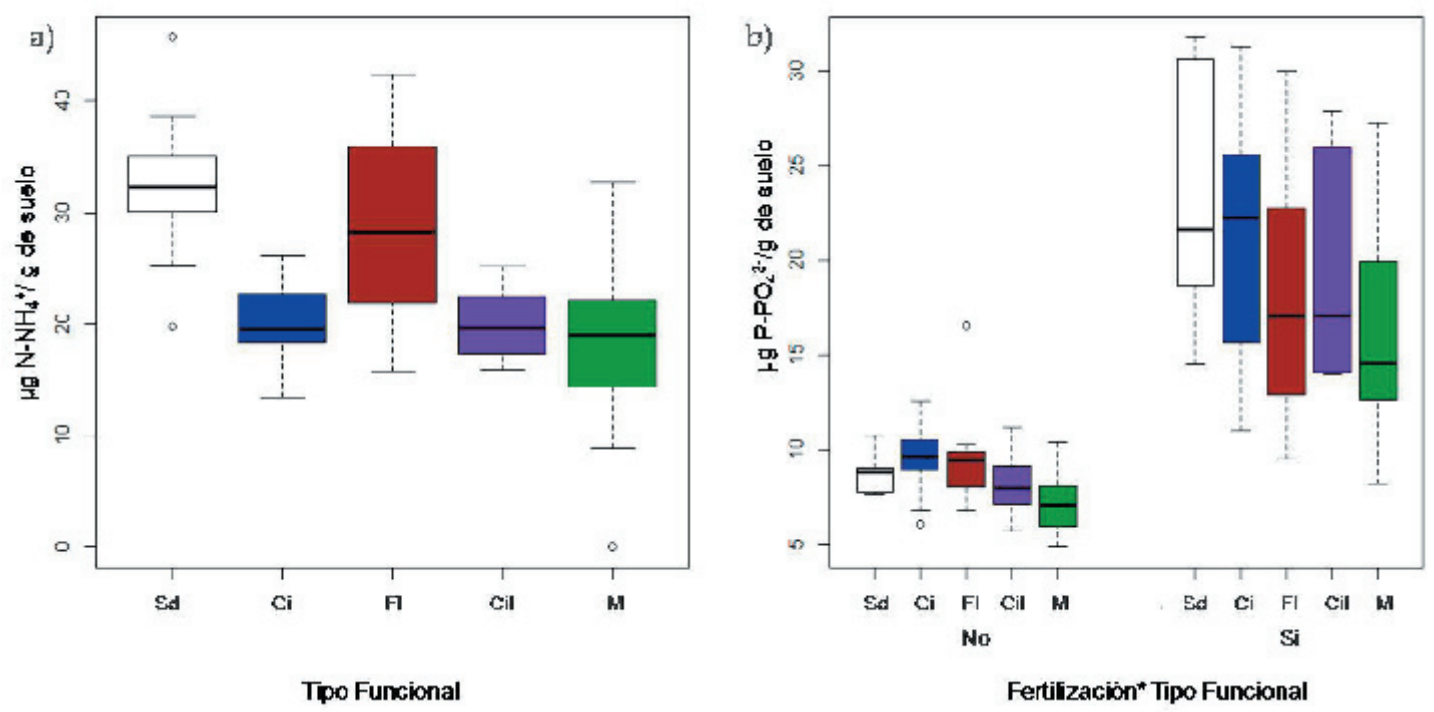

Figura 4. Concentración de a) $\mathrm{N}-\mathrm{NH}_{4}{ }^{+}$y b) $\mathrm{P}_{-} \mathrm{PO}_{4}{ }^{3-}$ en suelos con diferentes tipos funcionales de costras biológicas del suelo, CBS (Ci: cianobacterias; Fl: ficolíquenes; Cil: cianolíquenes; M: musgos) y suelo desnudo (Sd).

Figure 4. Concentration of a) $\mathrm{N}-\mathrm{NH}_{4}{ }^{+}$and b) $\mathrm{P}_{-}-\mathrm{PO}_{4}{ }^{3-}$ in soils with different functional types of biological soil crust, BSC (Ci: cyanobacterias; Fl: phycolichens; Cil: cyanolichens; M: mosses) and bare soil (Sd).

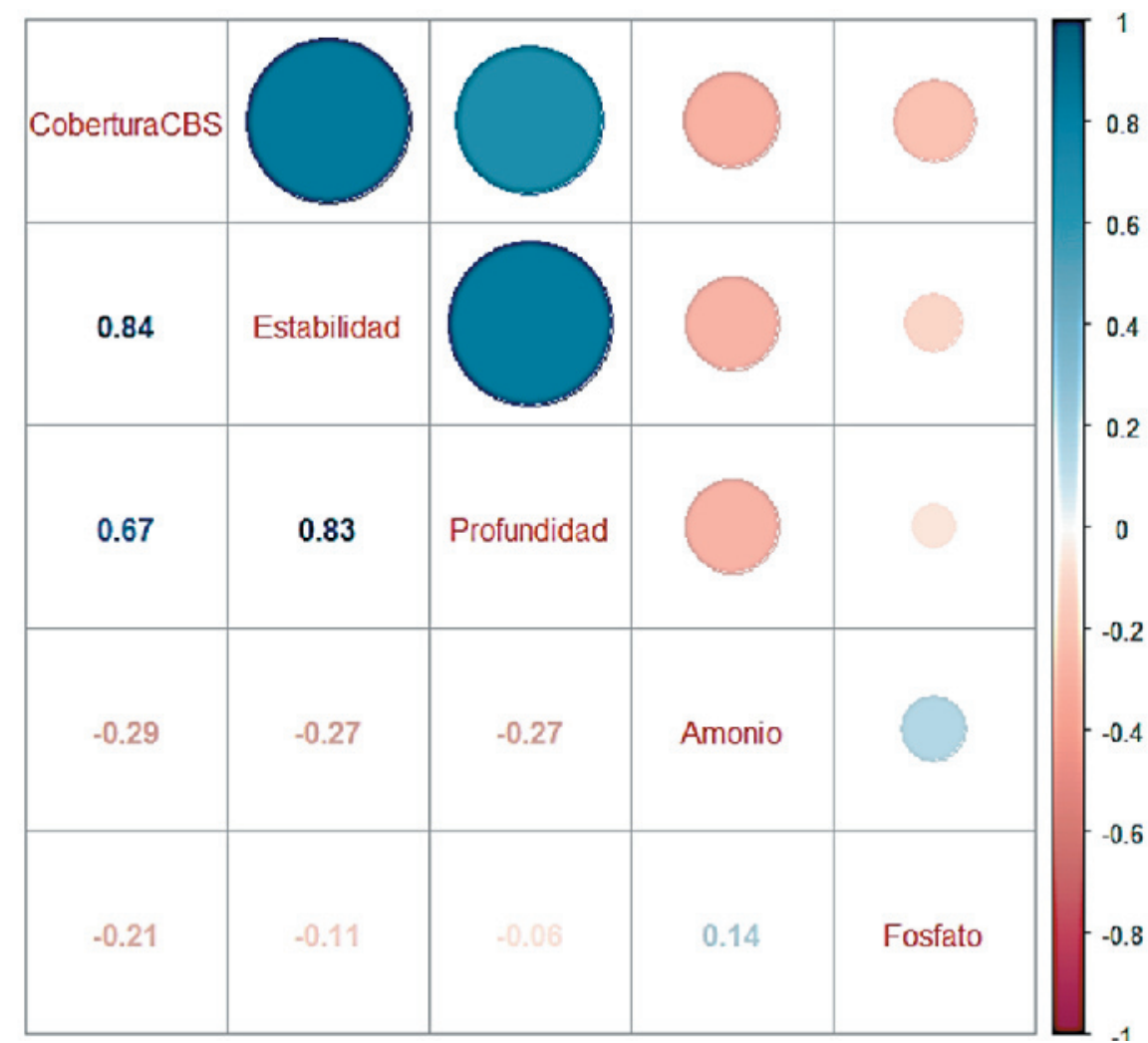

Figura 5. Matriz de correlación para las variables cuantitativas cobertura total de costras biológicas (CBS), estabilidad del suelo, profundidad de CBS, amonio y fosfato. En la diagonal se detallan las variables; el tamaño de los círculos representa la magnitud de correlación ( 0 a 1) y la intensidad de color representa la dirección. Por debajo de la diagonal se observan los valores de correlación $r$.

Figure 5. Correlation matrix for quantitative variables total biological soil crust cover, soil stability, BSC depth, ammonium and phosphate. The diagonal shows the variables; circle sizes represent correlation magnitude ( 0 to 1$)$ and color represents direction (blue is positive, peach is negative). Below the diagonal, the correlation values $(r)$ are presented. 
sembradas con todos los tipos funcionales de CBS presentaron concentraciones de amonio menores que el suelo desnudo (Tabla 5, Figura 4). Para las concentraciones de fosfato, el modelo que mejor ajustó los datos incluyó tipo funcional y fertilización (Tabla 1). La fertilización aumentó la concentración de fosfato (Tabla 5). Con respecto al tipo funcional sembrado, las mayores concentraciones de fosfato se registraron en suelo desnudo (macetas control) y las menores concentraciones en musgos (Figura 4).

\section{Correlación entre variables respuestas}

La estabilidad y la profundidad de CBS se relacionaron positivamente con la cobertura total de CBS. La mayor correlación resultó entre la cobertura total de CBS y la estabilidad del suelo ( $r=0.84)$. Los nutrientes del suelo se relacionaron negativamente con la cobertura, profundidad y estabilidad (Figura 5).

\section{Discusión}

En esteestudio se lograron propagar conéxito, en invernadero, diferentes tipos funcionales de organismos que componen la costra biológica del suelo (CBS) del Monte. La recuperación natural de la costra biológica puede tardar más de 40 años, sin embargo su inoculación en campo o su cultivo en invernadero acelera los tiempos de recuperación (Belnap 1993; Antoninka et al. 2016; Antoninka et al. 2018). Asimismo, los resultados aquí presentados muestran que en 19 meses las CBS alcanzaron coberturas hasta 5 veces mayores en macetas inoculadas que en aquellas sin inocular. Consistente con la hipótesis planteada, la desagregación de organismos aumentó la cobertura total y profundidad de la CBS y la estabilidad al suelo. Sin embargo, el agregado de fertilizante natural aumentó levemente la cobertura total de algunos tipos funcionales y disminuyó otras, y tuvo efecto nulo sobre la profundidad y estabilidad del suelo. El contenido de nutrientes dependió del tipo funcional y la concentración en el suelo se relacionó negativamente con la cobertura total de CBS.

Al finalizar el ensayo aumentó el número de tipos funcionales presentes en cada maceta (Figura 2; ver Material Suplementario Figura 1), coincidente con ensayos en invernáculo donde se inocularon costras de musgos provenientes del desierto occidental de Utah, y luego de 6 meses crecieron además cianobacterias y líquenes (Antoninka et al. 2016). Las cianobacterias, cianolíquenes y los musgos crecieron en macetas sembradas con todos los tipos de CBS, pero los últimos fueron los que alcanzaron mayores coberturas y en menor tiempo (observación personal). Además, la mayoría de las macetas inoculadas con musgos presentaron coberturas mayores al 30\%. La presencia de musgos en todos los tipos de macetas podría deberse al sombreado del invernadero, que replica la radiación encontrada bajo arbustos en Ñacuñán, donde los musgos son más abundantes (Gómez et al. 2012). Además, el riego por capilaridad mantiene la humedad del suelo, lo que beneficia a musgos que tienen alta demanda de agua (Antoninka et al. 2016; Tamm et al. 2018). Por el contrario, las cianobacterias y los cianolíquenes son capaces de crecer tanto en ambientes de elevada radiación y menor humedad que los musgos (Büdel et al. 1997; Mager and Thomas 2011) como en espacios abiertos entre la vegetación (Gómez et al. 2012). El alto contenido de agua puede limitar la capacidad fotosintética de los líquenes mediante suprasaturación, debido a que en las mismas cavidades intercambian el dióxido de carbono y el agua (Lange et al. 2001; Tamm et al. 2018). Al final del experimento, durante períodos secos y menor humedad relativa (Servicio Meteorológico Nacional 2018) pudo observarse la recuperación y crecimiento de algunos talos de líquenes, aunque no se midieron sistemáticamente y deberá abordarse en futuros ensayos.

La CBS desarrollada al concluir el ensayo bastó para brindar estabilidad al suelo, que fue mayor en macetas inoculadas que en los controles y varió según los organismos dominantes. Estas mejoras en las propiedades del suelo pueden deberse a exopolisacáridos de cianobacterias, y a estructuras como ricinas, rizohifas de líquenes y rizoides de musgos, que les permiten anclarse a mayor profundidad y unirse a partículas de suelo, generando agregados más resistentes a la erosión (Bowker et al. 2008; Weber et al. 2016). Con la siembra triturada aumentó la estabilidad del suelo y profundidad de las costras, lo que posiblemente esté relacionado a mayores coberturas totales alcanzadas con este tipo de siembra. Por lo tanto, luego de 19 meses la cobertura de CBS se relacionó de forma positiva con la estabilidad de suelos.

La mayor concentración de nutrientes se obtuvo en suelos desnudos, lo que coincide con ensayos realizados en pastizales semiáridos 
del oeste de Montana, donde parcelas inoculadas con musgos y líquenes tuvieron menos amonio que las control (Slate et al. 2019). La disminución de N y P puede deberse a la absorción de los mismos por las CBS, para aumento de biomasa y exopolisacáridos, que otorgaron mayor estabilidad al suelo, como ocurrió con CBS del desierto de Chihuaha, Nuevo México, que incorporan estos nutrientes para su desarrollo, evitando que queden retenidos en el sustrato (Crain et al. 2018). Las macetas con menor concentración de nutrientes fueron las sembradas con musgos que registraron mayores coberturas y alcanzaron mayor profundidad y biomasa, por el desarrollo de rizoides y estructuras aéreas. Probablemente, a mayor tiempo de desarrollo de las CBS, los organismos que la componen comiencen a liberar $\mathrm{N}$ al suelo por mineralización, lixiviación, muerte o recambio de algunos organismos. Sin embargo, hay casos de ensayos de invernadero, como por ejemplo el cultivo de costras de musgos del desierto de Utah, donde en tan sólo 6 meses se obtuvieron CBS funcionalmente maduras (Antoninka et al. 2016). El estiércol de cabra es un fertilizante natural que aporta al suelo nutrientes como nitrógeno y fósforo (Irshad et al. 2013). En el presente estudio, la fertilización no influyó en la concentración de amonio en el suelo, tal vez por la presencia de otros procesos que afectan las concentraciones de amonio, como la fijación de $\mathrm{N}$ atmosférico por ciertos microorganismos y la volatilización (McCalley and Sparks 2008). Por su parte, la contribución extra de fosfato fue dada principalmente por el estiércol de cabra, lo que resultó en mayor concentración de este nutriente en suelos fertilizados que sin fertilizar, aunque la fertilización no aumentó el desarrollo de las CBS.

Implementar tratamientos apropiados de fertilización, siembra y riego es esencial para el cultivo exitoso de organismos. En ensayos de invernáculo con costras de líquenes y cianobacterias, extraídas de arbustales del sureste de España, el agregado de fertilizantes orgánicos aceleró la propagación de las costras (Maestre et al. 2006). Por el contrario, en el presente estudio, la fertilización tuvo efecto negativo sobre la cobertura de ficolíquenes y musgos (Tabla 3; ver Material Suplementario Figura 2). La siembra triturada benefició a los musgos, tal vez por su capacidad de reproducirse asexualmente desde cualquier fragmento. Además, estos organismos producen protonema, filamentos fotosintéticos que se propagan paralelos al sustrato, desde los cuales se puede desarrollar un nuevo individuo (Frey and Kürschner 2011; Whitehouse 1980). En macetas inoculadas con ficolíquenes, la siembra triturada también favoreció el aumento de cobertura de CBS, aunque los organismos desarrollados fueron principalmente musgos. La costra dominada por ficolíquenes parece funcionar como reservorio de otros organismos de CBS.

En macetas donde se sembraron líquenes triturados se registró mayor cobertura total de CBS que en aquellas con siembra en trozos. De esta manera se favoreció la reproducción por fragmentación. Este proceso consiste en la ruptura del talo del liquen a fragmentos que contienen células del ficobionte y del micobionte, y la posterior dispersión, y es común, por ejemplo, en áreas pastoreadas del noreste de Alemania (Heinken 1999). En algunas macetas tanto de siembra triturada como en trozos, crecieron inicialmente cianobacterias o algas libres que formaron colonias, y luego se desarrollaron en líquenes gelatinosos. Al triturar los cianolíquenes podría haberse modificado la simbiosis entre la cianobacteria y el hongo, separando ambos organismos. La formación de un talo, por medio de la asociación entre la cianobacteria y el hongo, puede ser un proceso lento, desde 6 meses hasta dos años (Yoshimura et al. 1993) y requiere de condiciones ambientales favorables de humedad y radiación. Además, cada especie de hongo es altamente selectiva en su elección de ficobiontes, y éstos no siempre se encuentran libres en el sustrato (Ahmadjian and Jacobs 1981; Sanders and Lücking 2002). Los talos se formaron a los tres meses del inicio del experimento en macetas inoculadas con líquenes y en algunas macetas inoculadas con cianobacterias en las que se desarrollaron pequeños talos de cianolíquenes sin estructuras reproductivas sexuales.

Se confirma, entonces, que la CBS es una comunidad compleja integrada por varios organismos, difícilmente aislados unos de otros, que responden de forma diferente a las condiciones ambientales y los cuales interactúan cumpliendo diferentes funciones. Es necesario profundizar en la identificación taxonómica de géneros y especies que fueron exitosas, para diferenciar las funciones ecosistémicas que cumplen los organismos (Pietrasiak et al. 2013) y aplicarlos en ensayos de restauración.

En estudios anteriores sobre la CBS del monte en Mendoza se encontró que las CBS dominadas por líquenes presentaban 
la mayor cobertura y que estos grupos funcionales se distribuían principalmente en el borde de arbustos o en espacios abiertos entre arbustos. Por el contario, los musgos presentaron menor cobertura y se encontraron en espacios sombreados asociados a arbustos, predominantemente Larrea sp. (Gómez et al. 2012; García et al. 2015). Para este estudio, la recolección de muestras se realizó en picadas o en márgenes de las mismas en las que se removió la vegetación y la radiación es mayor, por lo que fueron más abundantes cianobacterias y cianolíquenes. Además, es importante resaltar que los suelos sin inocular en el ensayo también tuvieron presencia de cianobacterias y musgos, indicando que el suelo utilizado como sustrato y extraído de la Reserva de Nacuñán es un reservorio de estas comunidades. Podrían, por lo tanto, aplicarse estrategias de restauración pasiva en campos aledaños a esta zona, aunque teniendo en cuenta que, tal vez, la recuperación de la CBS sería más lenta que con inoculación o trasplante de estas comunidades.

Se ha sugerido que la inoculación de cianobacterias es un método efectivo para restaurar suelos en desiertos de China. Al colocar estos microorganismos, se prepara el suelo para facilitar el desarrollo de otros organismos como líquenes y musgos, y por consiguiente se recuperan las CBS del suelo (Wang et al. 2009). Asimismo, el cultivo de cianobacterias en cámaras de crecimiento con diferentes condiciones para la aclimatación gradual de los organismos acelera las tasas de establecimiento y recuperación en condiciones de campo (Giraldo-Silva et al. 2019). En el presente trabajo, los resultados demuestran que cuando se inocularon cianobacterias, éstas no tuvieron éxito en la propagación, coincidiendo con muestreos in situ que indican la poca cobertura de CBS dominadas por cianobacterias en el desierto de Mendoza (García et al. 2015).

Si bien los musgos alcanzaron mayor cobertura, tanto cianobacterias como cianolíquenes estuvieron presentes en las costras dominadas por los otros tipos funcionales. Por lo tanto, y de acuerdo con los resultados, se recomienda cultivar o trasplantar a suelos degradados una combinación de estos organismos en forma triturada, método que favorece la dispersión y establecimiento de algunas especies (Rosentreter 2020).

Los ecosistemas del Monte están sufriendo procesos de degradación que se caracterizan por la pérdida de vegetación y suelos (Villagra et al. 2009). Este trabajo aporta conocimiento sobre los posibles impactos de las CBS en el aumento de cobertura y estabilidad de suelos, ya que son pioneras enáreas dondeeldesarrollo de las plantas vasculares es limitado, como en suelos degradados, o ambientes de extrema aridez. Esta información permite considerar a las CBS como indicadores de degradación y promotoras de recuperación. Además, las CBS pueden colonizar espacios entre parches de vegetación formando comunidades que contribuyen a la multifuncionalidad del ecosistema (Garibotti et al. 2018). Por lo tanto, podrían plantearse proyectos de manejo, conservación o restauración que incluyan en conjunto a plantas vasculares y a los distintos grupos funcionales de la CBS para lograr mayores coberturas, diversidad y resistencia a la erosión en suelos de ecosistemas áridos.

\section{Conclusiones}

Nuestros resultados indican que el cultivo en invernadero de diferentes tipos funcionales favoreció la cobertura y la profundidad de las costras biológicas del suelo (CBS), y la estabilidad de suelos. Debido a este aumento de cobertura, la concentración de nutrientes disminuyó en suelos inoculados en comparación con suelos desnudos. Las CBS trituradas favorecieron el desarrollo en macetas sembradas con musgos y ficolíquenes, y la adición de fertilizante tuvo efecto negativo para estos organismos. Además, al finalizar el ensayo aumentó el número de tipos funcionales presentes en las macetas. Los musgos estuvieron presentes en todas las macetas y alcanzaron mayor cobertura con respecto al resto de los tipos funcionales, lo cual indica su potencial de recuperación. Estos resultados incrementan el conocimiento para el diseño de estrategias que involucren a la CBS como herramienta en la restauración activa y pasiva de tierras áridas del Monte.

Agradecimientos. Los autores agradecen al Técnico principal Marcelo Bourguet, Técnico Principal Gualberto Zalazar, al Profesional Asistente Hugo Debandi, Técnico Cristian Sancho y al Dr. Sebastián Crespo por la asistencia en el mantenimiento del ensayo. A la Dra. María Elena Corvalán por la colaboración en la colecta de material y al Lic. Ricardo Elia Dazat por la ayuda con los análisis químicos. A los Dres. Paul DeLey y Nicole Pietrasiak por donar material para los ensayos, como los kits de estabilidad de suelo. A la Dra. Carla Giordano y Lic. Lucía Biruk por préstamo 
y asesoramiento con el instrumental para medir radiación. Este proyecto fue financiado por subsidios otorgados a J. Aranibar por
ANPCyT (PICT 2015-0582), y SIIP (Secretaría de Investigación, Internacionales y Posgrado), UNCuyo, Mendoza.

\section{REFERENCIAS}

Ahmadjian, V., and J. B. Jacobs. 1981. Relationship between fungus and alga in the lichen Cladonia cristatella Tuck. Nature 289:169. https://doi.org/10.1038/289169a0.

Antoninka, A., M. A. Bowker, P. Chuckran, N. N. Barger, S. Reed, and J. Belnap. 2018. Maximizing establishment and survivorship of field-collected and greenhouse-cultivated biocrusts in a semi-cold desert. Plant and Soil 429:213-225. https://doi.org/10.1007/s11104-017-3300-3.

Antoninka, A., M. A. Bowker, S. C. Reed, and K. Doherty. 2016. Production of greenhouse grown biocrust mosses and associated cyanobacteria to rehabilitate dryland soil function. Restoration Ecology 24:324-335. https://doi.org/ 10.1111/rec.12311.

Aranibar, J. N., I. C. Anderson, S. Ringrose, and S. A. Macko. 2003. Importance of nitrogen fixation in soil crusts of southern African arid ecosystems: acetylene reduction and stable isotope studies. Journal of Arid Environments 54: 345-358. https://doi.org/10.1006/jare.2002.1094.

Barton K. 2018. MuMIn: Multi-Model Inference. R package version 1.42.1. URL: cran.r-project.org/package=MuMIn.

Belnap, J. 1993. Recovery rates of cryptobiotic crusts: inoculant use and assessment methods. Great Basin Naturalist 53: 89-95.

Belnap, J. 2002. Nitrogen fixation in biological soil crusts from southeast Utah, USA. Biology and Fertility of Soils 35: 128-135. https://doi.org/10.1007/s00374-002-0452-x.

Belnap, J., and O. L. Lange. 2001. Biological soil crusts: Structure, function, and management. Springer-Verlag, Berlin, Heidelberg.

Bessega, C., M. Cony, B. O. Saidman, R. Aguiló, P. E. Villagra, J. A. Álvarez, C. Pometti, and J. C. Vilardi. 2019. Genetic diversity and differentiation among provenances of Prosopis flexuosa DC (Leguminosae) in a progeny trial: Implications for arid land restoration. Forest Ecology and Management 443:59-68. https://doi.org/10.1016/j.foreco.2019.04.016.

Bowker, M. A., J. Belnap, V. B. Chaudhary, and N. C. Johnson. 2008. Revisiting classic water erosion models in drylands: the strong impact of biological soil crusts. Soil Biology and Biochemistry 40:2309-2316. https://doi.org/ 10.1016/j.soilbio.2008.05.008.

Bowker, M. A., J. Belnap, and M. E. Miller. 2006. Spatial modeling of biological soil crusts to support rangeland assessment and monitoring. Rangeland Ecology and Management 59:519-529. https://doi.org/10.2111/05-179R1.1.

Bowker, M. A., R. L. Mau, F. T. Maestre, C. Escolar, and A. P. Castillo'Monroy. 2011. Functional profiles reveal unique ecological roles of various biological soil crust organisms. Functional Ecology 25:787-795. https://doi.org/10.1111/ j.1365-2435.2011.01835.x.

Büdel, B., U. Karsten, and F. García-Pichel. 1997. Ultraviolet-absorbing scytonemin and mycosporine-like amino acid derivatives in exposed, rock-inhabiting cyanobacterial lichens. Oecologia 112:165-172. https://doi.org/10.1007/ s004420050296.

Cabrera, A. L. 1976. Regiones fitogeográficas argentinas. Enciclopedia Argentina de Agricultura y Jardinería (2da. Ed.). Tomo II, Fase 1 ACME, Buenos Aires. Pp. 85.

Casas, C., M. Brugués, R. M. Cros, and C. Sérgio. 2006. Handbook of Mosses of the Iberian Peninsula and the Balearic Islands. Institut d'Estudis Catalans, Barcelona.

Chiquoine, L. P., S. R. Abella, and M. A. Bowker. 2016. Rapidly restoring biological soil crusts and ecosystem functions in a severely disturbed desert ecosystem. Ecological Applications 26:1260-1272. https://doi.org/10.1002/15-0973.

Corvalán Videla, M. E., M. D. L. A. Taboada, and J. N. Aranibar. 2018. Diversidad de cianobacterias en costras biológicas de suelo de la ecorregión del Monte Central (Mendoza, Argentina). Lilloa 55:30-46. https://doi.org/10.30550/j.lil/ 2018.55.2/4.

Crain, G., J. McLaren, B. Brunner, and A. Darrouzet-Nardi. 2018. Biologically Available Phosphorus in BiocrustDominated Soils of the Chihuahuan Desert. Soil Systems 2:56. https://doi.org/10.3390/soilsystems2040056.

Dalmasso, A. D. 2010. Revegetación de áreas degradadas con especies nativas. Boletín de la Sociedad Argentina de Botánica 45:149-171.

Delignette-Muller, M. L., and C. Dutang. 2015. fitdistrplus: An R Package for Fitting Distributions. Journal of Statistical Software 64(4):1-34. URL: http://www.jstatsoft.org/v64/i04/. https://doi.org/10.18637/jss.v064.i04.

Eldridge, D. J., and J. F. Leys. 2003. Exploring some relationships between biological soil crusts, soil aggregation and wind erosion. Journal of Arid Environments 53:457-466. https://doi.org/10.1006/jare.2002.1068.

Eldridge, D. J., D. Freudenberger, and T. B. Koen. 2006. Diversity and abundance of biological soil crust taxa in relation to fine and coarse-scale disturbances in a grassy eucalypt woodland in eastern Australia. Plant and Soil 281:255-268. https://doi.org/10.1007/s11104-005-4436-0.

Fernández, M. E., C. B. Passera, and M. A. Cony. 2016. Sapling growth, water status and survival of two native shrubs from the Monte Desert, Mendoza, Argentina, under different preconditioning treatments. Revista de la Facultad de Ciencias Agrarias 48:110-125.

Frey, W., and H. Kürschner. 2011. Asexual reproduction, habitat colonization and habitat maintenance in bryophytes. Flora-Morphology, Distribution, Functional Ecology of Plants 206:173-184. https://doi.org/10.1016/ 
j.flora.2010.04.020.

Gao, L., M. A. Bowker, M. Xu, H. Sun, D. Tuo, and Y. Zhao. 2017. Biological soil crusts decrease erodibility by modifying inherent soil properties on the Loess Plateau, China. Soil Biology and Biochemistry 105:49-58. https://doi.org/10.1016/ j.soilbio.2016.11.009.

Garibotti, I. A., M. González Polo, and S. Tabeni. 2018. Linking biological soil crust attributes to the multifunctionality of vegetated patches and interspaces in a semiarid shrubland. Functional Ecology 32:1065-1078. https://doi.org/ $10.1111 / 1365-2435.13044$

García, V. R., J. N. Aranibar, and N. Pietrasiak. 2015. Multiscale effects on biological soil crusts cover and spatial distribution in the Monte Desert. Acta Oecologica 69:35-45. https://doi.org/10.1016/j.actao.2015.08.005.

Gichangi, E. M., and P. N. S. Mnkeni. 2009. Effects of goat manure and lime addition on phosphate sorption by two soils from the Transkei Region, South Africa. Communications in Soil Science and Plant Analysis 40:3335-3347. https: //doi.org/10.1080/00103620903325943.

Giraldo'Silva, A., C. Nelson, C. Penfold, N. N. Barger, and F. García'Pichel. 2019. Effect of preconditioning to the soil environment on the performance of 20 cyanobacterial cultured strains used as inoculum for biocrust restoration. Restoration Ecology. https://doi.org/10.1111/rec.13048.

Goirán, S. B., J. N. Aranibar, and M. L. Gómez. 2012. Heterogeneous spatial distribution of traditional livestock settlements and their effects on vegetation cover in arid groundwater coupled ecosystems in the Monte desert (Argentina). Journal of Arid Environments 87:188-197. https://doi.org/10.1016/j.jaridenv.2012.07.011.

Gómez, D. A., J. N. Aranibar, S. Tabeni, P. E. Villagra, I. A. Garibotti, and A. Atencio. 2012. Biological soil crust recovery after long-term grazing exclusion in the Monte Desert (Argentina). Changes in coverage, spatial distribution, and soil nitrogen. Acta Oecologica 38:33-40. https://doi.org/10.1016/j.actao.2011.09.001.

Heinken, T. 1999. Dispersal patterns of terricolous lichens by thallus fragments. The Lichenologist 31:603-612. https: //doi.org/10.1017/S0024282999000791. https://doi.org/10.1006/lich.1999.0219.

Herrick, J. E., W. G. Whitford, A. G. De Soyza, J. W. Van Zee, K. M. Havstad, C. A. Seybold, and M. Walton. 2001. Field soil aggregate stability kit for soil quality and rangeland health evaluations. Catena 44:27-35. https://doi.org/ 10.1016/S0341-8162(00)00173-9.

Irshad, M., A. E. Eneji, Z. Hussain, Z., and M Ashraf. 2013. Chemical characterization of fresh and composted livestock manures. Journal of Soil Science and Plant Nutrition 13:115-121. https://doi.org/10.4067/S0718-95162013005000011.

Komárek, J., and J. R. Johansen. 2015. Filamentous cyanobacteria. In Freshwater Algae of North America . Academic Press. https://doi.org/10.1016/B978-0-12-385876-4.00004-9.

Kröpfl, A. I., V. A. Deregibus, and G. A. Cecchi. 2007. Disturbios en una estepa arbustiva del Monte: cambios en la vegetación. Ecología Austral 17:257-268.

Lange, O. L., T. A. Green, and U. Heber. 2001. Hydration-dependent photosynthetic production of lichens: what do laboratory studies tell us about field performance? Journal of Experimental Botany 52:363. https://doi.org/10.1093/ jexbot/52.363.2033.

Maestre, F. T., N. Martín, B. Díez, R. Lopez-Poma, F. Santos, I. Luque, and J. Cortina. 2006. Watering, fertilization, and slurry inoculation promote recovery of biological crust function in degraded soils. Microbial Ecology 52:365-377. https://doi.org/10.1007/s00248-006-9017-0.

Mager, D. M., and A. D. Thomas. 2011. Extracellular polysaccharides from cyanobacterial soil crusts: a review of their role in dryland soil processes. Journal of Arid Environments 75:91-97. https://doi.org/10.1016/j.jaridenv.2010.10.001.

Mallen-Cooper, M., and D. J. Eldridge. 2016. Laboratory-based techniques for assessing the functional traits of biocrusts. Plant and Soil 406:131-143. https://doi.org/10.1007/s11104-016-2870-9.

McCalley, C. K., and J. P. Sparks. 2008. Controls over nitric oxide and ammonia emissions from Mojave Desert soils. Oecologia 156:871-881. https://doi.org/10.1007/s00442-008-1031-0.

Mupondi, L. T., P. N. S. Mnkeni, and M. O. Brutsch. 2006. The effects of goat manure, sewage sludge and effective microorganisms on the composting of pine bark. Compost Science and Utilization 14:201-210. https://doi.org/10.1080/ 1065657X.2006.10702284.

Nash III, T. H., B. D. Ryan, C. Gries, and F. Bungartz. 2002. Lichen Flora of the Greater Sonora Desert Region, vol. 1. Lichen Unlimited, Tempe, Arizona, USA.

Nash III, T. H., B. D. Ryan, P. Diederich, C. Gries, and F. Bungartz. 2001. Lichen Flora of the Greater Sonora Desert Region, vol 2. Lichen Unlimited, Tempe, Arizona, USA.

Nash III, T. H., C. Gries, and F. Bungartz. 2001. Lichen Flora of the Greater Sonora Desert Region, vol. 3. Lichen Unlimited, Tempe, Arizona, USA.

Okalebo, J. R., K. W. Gathua, and P. L. Woomer. 1993. Laboratory methods of soil and plant analysis: A Working Manual. Second edition. Sacred Africa, Nairobi, Kenya.

Pietrasiak, N., J. U. Regus, J. R. Johansen, D. Lam, J. L. Sachs, and L. S. Santiago. 2013. Biological soil crust community types differ in key ecological functions. Soil Biology and Biochemistry 65:168-171. https://doi.org/10.1016/ j.soilbio.2013.05.011.

Read, C. F., D. H. Duncan, P. A. Vesk, and J. Elith. 2014. Biocrust morphogroups provide an effective and rapid assessment tool for drylands. Journal of Applied Ecology 51:1740-1749. https://doi.org/10.1111/1365-2664.12336.

RStudio Team. 2015. RStudio: Integrated Development for R. RStudio, Inc., Boston, MA. URL: http://www.rstudio.com/

Rosentreter, R. 2020. Biocrust lichen and moss species most suitable for restoration projects. Restoration Ecology 28 : 
67-74. https://doi.org/10.1111/rec.13082.

Sanders, W. B., and R. Lücking. 2002. Reproductive strategies, relichenization and thallus development observed in situ in leaf'dwelling lichen communities. New Phytologist 155:425-435. https://doi.org/10.1046/j.1469-8137.2002.00472.x.

Sartor, C. E. 2015. Influencia de Prosopis flexuosa sobre el establecimiento de gramíneas perennes en dos sitios del Monte mendocino. Tesis de doctorado en Ciencias Biológicas. Universidad Nacional de Córdoba, Córdoba. Argentina. Pp. 139.

Segui, N. S. 2019. Técnicas de restauración en zonas áridas de Mendoza: supervivencia y crecimiento de plantines de Prosopis flexuosa y Prosopis chilensis con distinta época de trasplante y procedencia. Tesis de grado. Ingeniera en Recursos Naturales Renovables. Universidad Nacional de Cuyo, Mendoza. Argentina. Pp. 71.

Servicio Meteorológico Nacional. 2018. Estadísticas climáticas normales. Datos Meteorológicos horarios. URL: www.smn.gob.ar/descarga-de-datos. Consultado en Diciembre 2020.

Slate, M. L., R. A. Durham, and D. E. Pearson. 2019. Strategies for restoring the structure and function of lichen'moss biocrust communities. Restoration Ecology. https://doi.org/10.1111/rec.12996.

Su, Y. G., X. R. Li, Y. W. Cheng, H. J. Tan, and R. L. Jia. 2007. Effects of biological soil crusts on emergence of desert vascular plants in North China. Plant Ecology 191:11-19. https://doi.org/10.1007/s11258-006-9210-8.

Tamm, A., J. Caesar, N. Kunz, C. Colesie, H. Reichenberger, and B. Weber. 2018. Ecophysiological properties of three biological soil crust types and their photoautotrophs from the Succulent Karoo, South Africa. Plant and Soil 429: 127-146. https://doi.org/10.1007/s11104-018-3635-4.

Villagra, P. E., E. Cesca, J. Álvarez, F. Rojas, M. Bourguet, C. Rubio, and P. Mastrángelo. 2010. Anexo II - Documento de Ordenamiento de Bosques Nativos de la Provincia de Mendoza. Secretaría de Medio Ambiente. Dirección de Recursos Naturales Renovables. Gobierno de Mendoza. Pp. 65.

Villagra, P. E., G. E. Defossé, H. F. Del Valle, S. Tabeni, M. Rostagno, E. Cesca, and E. Abraham. 2009. Land use and disturbance effects on the dynamics of natural ecosystems of the Monte Desert. Implications for their management. Journal of Arid Environments 73:202-211. https://doi.org/10.1016/j.jaridenv.2008.08.002.

Villagra, P. E., and J. A. Álvarez. 2019. Determinantes ambientales y desafíos para el ordenamiento forestal sustentable en los algarrobales del Monte, Argentina. Ecología Austral 29:146-155. https://doi.org/10.25260/EA.19.29.1.0.752.

Wang, W., Y. Liu, D. Li, C. H, and B. Rao. 2009. Feasibility of cyanobacterial inoculation for biological soil crusts formation in desert area. Soil Biology and Biochemistry 41:926-929. https://doi.org/10.1016/j.soilbio.2008.07.001.

Weatherburn, M. W. 1967. Phenolehypochlorite reaction for determination of ammonia. Analytical Chemistry 39:971974. https://doi.org/10.1021/ac60252a045.

Weber, B., B. Büdel, and J. Belnap. 2016. Biological soil crusts: an organizing principle in drylands. Springer. Berlin, Heidelberg, New York. https://doi.org/10.1007/978-3-319-30214-0.

Whitehouse, H. L. K. 1980. The production of protonemal gemmae by mosses growing in deep shade. Journal of Bryology 11:133-138. https://doi.org/10.1179/jbr.1980.11.1.133.

Wei, T., and V. Simko. 2017. R package “corrplot": Visualization of a Correlation Matrix (Version 0.84). URL: github.com/ taiyun/corrplot.

Williams, A. J., B. J. Buck, and M. A. Beyene. 2012. Biological soil crusts in the Mojave Desert, USA: Micromorphology and pedogenesis. Soil Science Society of America Journal 76:1685-1695. https://doi.org/10.2136/sssaj2012.0021.

Wong, C. S., and W. K. Li. 1998. A note on the corrected Akaike information criterion for threshold autoregressive models. Journal of Time Series Analysis 19:113-124. https://doi.org/10.1111/1467-9892.00080.

Yoshimura, I., T. Kurokawa, Y. Yamamoto, and Y. Kinoshita. 1993. Development of lichen thalli in vitro. Bryologist 96(3):412-421. https://doi.org/10.2307/3243871.

Zhao, Y. G., M. X. Xu, and J. Belnap. 2010. Potential nitrogen fixation activity of different aged biological soil crusts from rehabilitated grasslands of the hilly Loess Plateau, China. Journal of Arid Environments 74:1186-1191. https: //doi.org/10.1016/j.jaridenv.2010.04.006.

Zhao, Y., and M. Xu. 2012. Runoff and soil loss from revegetated grasslands in the hilly Loess Plateau region, China: influence of biocrust patches and plant canopies. Journal of Hydrologic Engineering 18:387-393. https://doi.org/ 10.1061/(ASCE)HE.1943-5584.0000633. 